\title{
Recent Improvements to NRL's Portable Hyperspectral Imager for Low Light Spectroscopy ${ }^{1,23}$
}

Daniel R. Korwan

Naval Research Laboratory Code 7212

4555 Overlook Ave., S.W. Washington, D.C. 20375

Phone: (202) 404-1391

korwanconrl.navy.mil

Jeffrey Bowles

Naval Research Laboratory Code 7212

4555 Overlook Ave., S.W. Washington, D.C. 20375

Phone: (202) 404-1021

Jeffrey.bowles a.nrl.navy.mil

\author{
Curtiss O. Davis \\ Naval Research Laboratory \\ Code 7212 \\ 4555 Overlook Ave., S.W. \\ Washington, D.C. 20375 \\ Phone: (202) 767-9296 \\ Davisorsd.nrl.navy.mil \\ Paul Bisset
}

Florida Environmental Research Institute, 4807 Bayshore Blvd., Tampa, FL 33611

(813) 837-3374

pbissett(aflenvironmental.org

David Kohler

Florida Environmental Research Institute, 4807 Bayshore Blvd., Tampa, FL 33611

(813) 837-3374

dkohler(aflenvironmental.org

Wei Chen

Naval Research Laboratory Code 7212

4555 Overlook Ave., S.W. Washington, D.C. 20375

Phone: (202) 404-1391

chen(arsd.nrl.navy.mil

'U.S. government work not protected by U.S. Copyright.

2 IEEEAC paper \#1459, Updated November 2, 2002.

${ }^{3}$ This research was supported by the U. S. Office of Naval Research. 
Abstract- The Naval Research Laboratory has been developing the Portable Hyperspectral Imager for Low Light Spectroscopy (PHILLS) system for the last 8 years. The latest system is called the Ocean PHILLS and it was designed primarily for work in the coastal ocean area. The Navy is interested in the optical properties of the water particularly bathymetry, water clarity, bottom type, etc. In addition, the measured water properties are used as input into various predictive models. The pushbroom imaging system has a backside illuminated $\mathrm{CCD}$, for high $\mathrm{QE}$ in the blue, and an Offner designed spectrograph, for low image distortion. The system covers approximately 400 to 1000 $\mathrm{nm}$ with a band spacing of $1.2,4.6$ or $9.2 \mathrm{~nm}$ depending on settings. Ground positioning is accomplished by using an integrated GPS/LNS system. The systems are designed using commercial off-the-shelf components in order to be relatively inexpensive. Because of this several of these systems are now in use by the Navy and are flown on a variety of aircraft for a range of applications. This paper highlights recent improvements in the instruments and instrument performance, and discusses some of the recent experiments and future planned deployments.

\section{TABLE OF CONTENTS}

1. INTRODUCTION

2. IMPROVEMENTS
a. CONTROL OF STRAY LIGHT
b. MODELING
c. TIME TAGGING

3. CONCLUSIONS (DEPLOYMENTS)

\section{INTRODUCTION}

Over the past eight years the Naval Research Laboratory has built a series of PHILLS hyperspectral imagers. Bowles, et al. [1] describes three of the earlier PHILLS cameras and their calibration and characterization. Davis, et. el. [2] describe a more recent incarnation, which was deployed at the LEO-15 site in 2001. Since 1994, PHILLS has undergone a series of design changes aimed at increasing signal to noise, stability, spatial and spectral resolution and swaths, while keeping construction costs down by utilizing commercial off-the-shelf parts. With each change calibration and characterization processes have been improved and there has been an increased understanding of the undesirable instrument artifacts, which has resulted in further design refinements and improved data processing. The purpose of this paper is to discuss the latest round of design improvements and their impact on recent deployments.

The PHILLS imaging spectrometer consists of essentially two independent optical systems: the fore-optics and slit, and the spectrometer and CCD camera. Typically the fore- optics is a Schneider Optics lens (Hauppauge, NY), aberration corrected from $400-1000 \mathrm{~nm}$, with a focal length of 12,17 , or $25 \mathrm{~mm}$ and operated at $\mathrm{F} / 4.0$. The lens images the scene down onto a 12 micron slit, which acts as a field stop. The HyperSpec ${ }^{\mathrm{TM}}$ spectrograph, designed collaboratively by the NRL and American Holographic, Inc. (now Agilent Technologies, Fitchburg, MA), re-images and disperses the slit onto a two-dimensional CCD camera. The design and specifications for the spectrograph are shown in figure 1. The Pluto ${ }^{\mathrm{TM}}$ camera is designed by PixelVision Inc. (Beaverton, OR), and incorporates a $1024 \times 1024$ pixel array with a 12 micron pixel pitch. The array is vacuum sealed and thermo-electrically cooled to lower dark noise and maintain stability. A standard Windows NT4.0 based personal computer controls low-level functions of the camera, including frame rate and analog gain, and a frame grabber is used to acquire each frame of data.

\begin{tabular}{|l|l|}
\hline HyperSpec & VS-15 Specifications \\
\hline Size & $180 \times 150 \times 150 \mathrm{~mm}$ \\
\hline Weight & $\begin{array}{l}24 \text { oz. (w/o camera } \\
\text { or lens) }\end{array}$ \\
\hline Field size & $12 \mathrm{~mm}$ \\
\hline Dispersion & $\begin{array}{l}400-1000 \mathrm{~nm} \text { over } 6 \\
\mathrm{~mm}\end{array}$ \\
\hline Aperture & $\mathrm{f} / 2$ \\
\hline Spot size & $<24 \mu \mathrm{m} \mathrm{rms}$ \\
\hline $\begin{array}{l}\text { Keystone } \\
\text { Distortion }\end{array}$ & $<0.1 \%$ \\
\hline $\begin{array}{l}\text { Smile } \\
\text { Distortion }\end{array}$ & $<0.1 \%$ \\
\hline
\end{tabular}

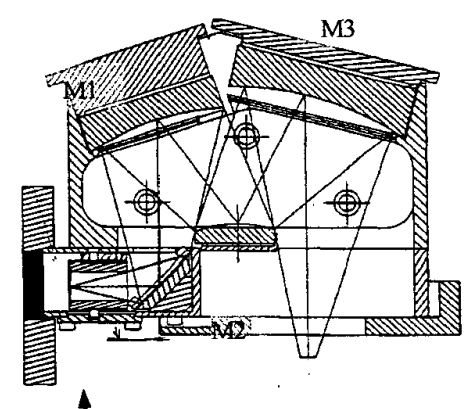

Figure 1. Desislit and specifications for the HyperSpec ${ }^{\mathrm{TM}}$ VS-15 Offner Spectrograph. M1, M2, and M3 are the primary, secondary, and tertiary mirrors of the Offner design. The dispersive element is the convex grating/mirror M2. The pictured rays originate at the slit, which is reimaged and dispersed at the focal plane (not shown).

Onboard the aircraft, the system is operated in a pushbroom-scanning mode, with the long dimension of the slit aligned to the crosstrack direction of the flight line. 
Thus the instantaneous (single frame) dispersed image of the slit on the array is divided into the spatial crosstrack direction and the spectral direction. The second spatial dimension of a full scene is built up of sequential instantaneous frames. Each full scene can be thought of has a hyperspectral data cube having two spatial dimensions and one spectral dimension. Geolocation of the data is provided by a global positioning satellite/inertial navigational system (GPS/INS) mounted next to the PHILLS. Each frame of the PHILLS data and the GPS/INS data are time tagged to allow them to be temporally matched during postprocessing.

\section{Improvements}

The previous incarnation of the PHILLS instrument, while producing high fidelity data [2], has since been modified. To preserve cost, it is built from commercial off-the-shelf parts. This presents a difficult challenge as one must make performance tradeoffs not required when one has the ability to design a unique instrument to study a specific scientific problem, in this case, characterization of the coastal environment. The current improvements are a result of small optical modifications of the previous instrument and better instrument modeling.

\section{Control of stray light}

Inherent in the HyperSpec ${ }^{\mathrm{TM}}$ Offner spectrograph is that light is diffracted by the grating into multiple orders that do not contribute to the primary first order signal. These unwanted orders must be accounted for by appropriate baffling or light dumps. For example, in deployments of the PHILLS prior to 2001, some of the zero-order portion of the diffraction pattern scattered inside the spectrometer and contaminated the data. One of the obvious sources of this scattering occurred off of a black anodized aluminum Cmount ring surrounding the camera window forming a distinct flaring pattern, with the centers of the zero-order peaks being closer to the center of the array at high channel numbers (longer wavelengths) than at low channel numbers. This pattern could be seen in across-sample profiles of the blue-wavelength channels of radiometric calibration data. While the effect was removed in post-processing it still limited the dynamic range of the of the affected channels and eventually a mask was placed on the outside window of the camera in order to block this light. One must also account for the unwanted second order diffracted light from short wavelengths, landing on the same $\mathrm{CCD}$ position as the first order light at longer wavelengths. To combat this effect an order sorting filter was placed on the outside of the camera window. The order sorter was a high pass filter that absorbs wavelengths shorter than $530 \mathrm{~nm}$ and was positioned in the path $580 \mathrm{~nm}-1000 \mathrm{~nm}$ first order light.

The placement of the filters was reasonably effective in controlling unwanted light. However, since they were positioned outside the camera window, the fore-optics were limited to a fresnel number 4.0 or slower. If the aperture of the fore-optics was opened any further, the light cone from the zero order light would overlap with the light from the blue channels, making it impossible to remove all of the zero order light without decreasing the signal in the blue. The margin for error on the positioning of the order sorter would be restrictive as well, for precisely the same reason.

As shown in figure 2, the data from the LEO-15 2001 deployment contained an additional stray light effect that occurs in the longer wavelength channels. While the exact cause of this problem is unknown, the presumed source is a reflection off of an anodized aluminum surface, which has a large specular reflectance at wavelengths longer than $700 \mathrm{~nm}$. The effect increases with wavelength both in the data and in the reflectance.

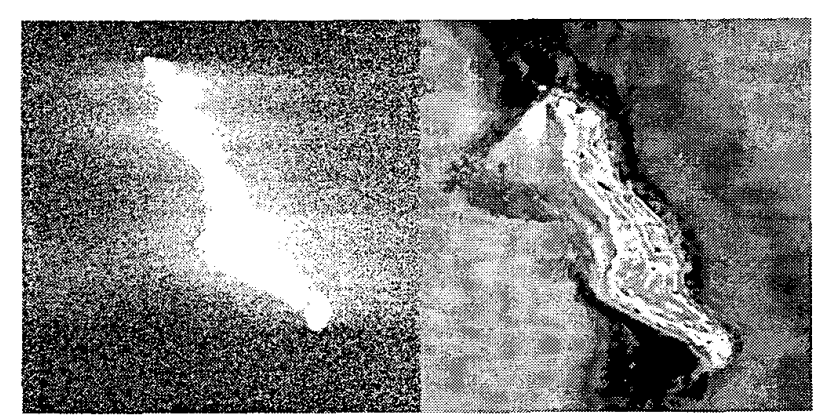

Figure 2. PHILLS 950nm channel image of an island at the LEO-15 site in 2002 showing light scattered from the red rich island into the red poor ocean (left). The same scene at the $530 \mathrm{~nm}$ channel showing no scatter (right). The flight line is in the vertical direction and the long axis of the slit in the horizontal direction. The images have been stretched to enhance the effect for the eye.

To alleviate these problems without making drastic changes to the system, the order sorter filter and zero order masks were moved inside the camera head, as close to the array as mechanically possible. In addition, most of the chip except for the active region was masked. All parts near the camera head and close to the optical path (including the zero order dump) were coated with Aeroglaze ${ }^{\mathrm{TM}} \mathrm{Z306}$, which is a good absorber across all of the PHILL's spectral channels. All coated pieces underwent a 24 hour vacuum bake out at $120 \mathrm{C}$ prior to assembly in order to remove all solvents and contaminants.

\section{Better Modeling}

Essential in the design of all optical remote sensing system is a good optical model of the system itself. It leads to a better understanding of how the instrument should work and expedites troubleshooting when problems are encountered (e.g. stray light). This is problematic given the commercial off-the-shelf parts approach because it is difficult to obtain accurate specifications of each part along the optical path, from entrance pupil to detector electronics. Fortunately we 
have been able to obtain Zemax ${ }^{\mathrm{TM}}$ prescriptions for the spectrograph and make reasonable assumptions on the Schneider lens (proprietary lens design) in order to have a relatively accurate modeling of the system through to the camera window. With the minor improvements made to the camera head, a reasonable model of the entire optical train has been constructed. This includes the coatings on the order sorting filter and the camera window, which were specified and the transmission measured by the NRL.

Several key results have been obtained with this better model. The first is simply the ability to provide better placement and tolerancing on the filters and masks, which in turn helps to control stray light. The second is that it is now known what is the expected spectral transmission through the system and key areas of vignetting within the spectrograph have been determined. Combined this knowledge allows one to identify the likely scattering sources of stray light, both spectral and spatial, and to better test these hypotheses.

However, the most important result is the determination of expected spot sizes at the focal plane array and how changes in the system effect these sizes. For example, the spectrograph must be manually focused to the array. Thus the depth of focus must be known in order to determine the best focus spot size and how it changes as one moves through focus. The biggest factors affecting the depth of focus are the fore-optics f-number, the width of the window and filter (spherical aberration becomes a factor when flat glass substrates are placed in a focused beam), and the telecentricity of the for-optics (the spectrograph is telecentric and a non telecentric lens will cause vignetting and aberrations at large field angles). It turns out that for very fast values of the f-number, it is actually impossible to get a spot size smaller than a pixel size (12 microns). Therefore, although placing the filters close to the array allows for faster f-numbers in terms of mechanical tolerancing as discussed earlier, there is still a fundamental limit to the f-number of the overall system. This effect is shown in figure 3 and table 1 . As seen in the table, at F/2.0 the spot sizes are much larger than the pixel size for most wavelengths and most field angles. F/4.0 has small spot sizes for virtually all wavelengths and field angles. Note that both the calculations in the figure and the table were performed for the model optimized at the best focus of the $400 \mathrm{~nm}$ channel.

\begin{tabular}{|c|c|c|c|}
\hline & \multicolumn{3}{|c|}{ Radial distance at given wavelength $(\mu \mathrm{m})$} \\
\hline Field & $400 \mathrm{~nm}$ & $700 \mathrm{~nm}$ & $1000 \mathrm{~nm}$ \\
\hline $0.00(\mathrm{~mm})$ & 5.07 & 9.34 & 12.93 \\
\hline $2.80(\mathrm{~mm})$ & 6.52 & 10 & 13.83 \\
\hline $5.36(\mathrm{~mm})$ & 11.48 & 11.83 & 16.13 \\
\hline
\end{tabular}

f/4.0

\begin{tabular}{|l|l|l|l|}
\hline & \multicolumn{3}{|c|}{ Radial distance at given wavelength $(\mu \mathrm{m})$} \\
\hline Field & $400 \mathrm{~nm}$ & $700 \mathrm{~nm}$ & $1000 \mathrm{~nm}$ \\
\hline $0.00(\mathrm{~mm})$ & 3.00 & 5.27 & 7.4 \\
\hline $2.80(\mathrm{~mm})$ & 3.82 & 5.22 & 7.48 \\
\hline $5.36(\mathrm{~mm})$ & 6.38 & 5.77 & 7.48 \\
\hline
\end{tabular}

Table 1. Radial distance in image space at which the total enclosed energy for a point object equals $90 \%$ of the total energy in the image. The values are presented for $f / 2.0$ and $\mathrm{f} / 4.0$, at 400,700 , and $1000 \mathrm{~nm}$, and for field points at the center, midpoint, and edge of the slit (field). The effects of the new window and OSF are included in the calculations. 
Vol. 4-1859

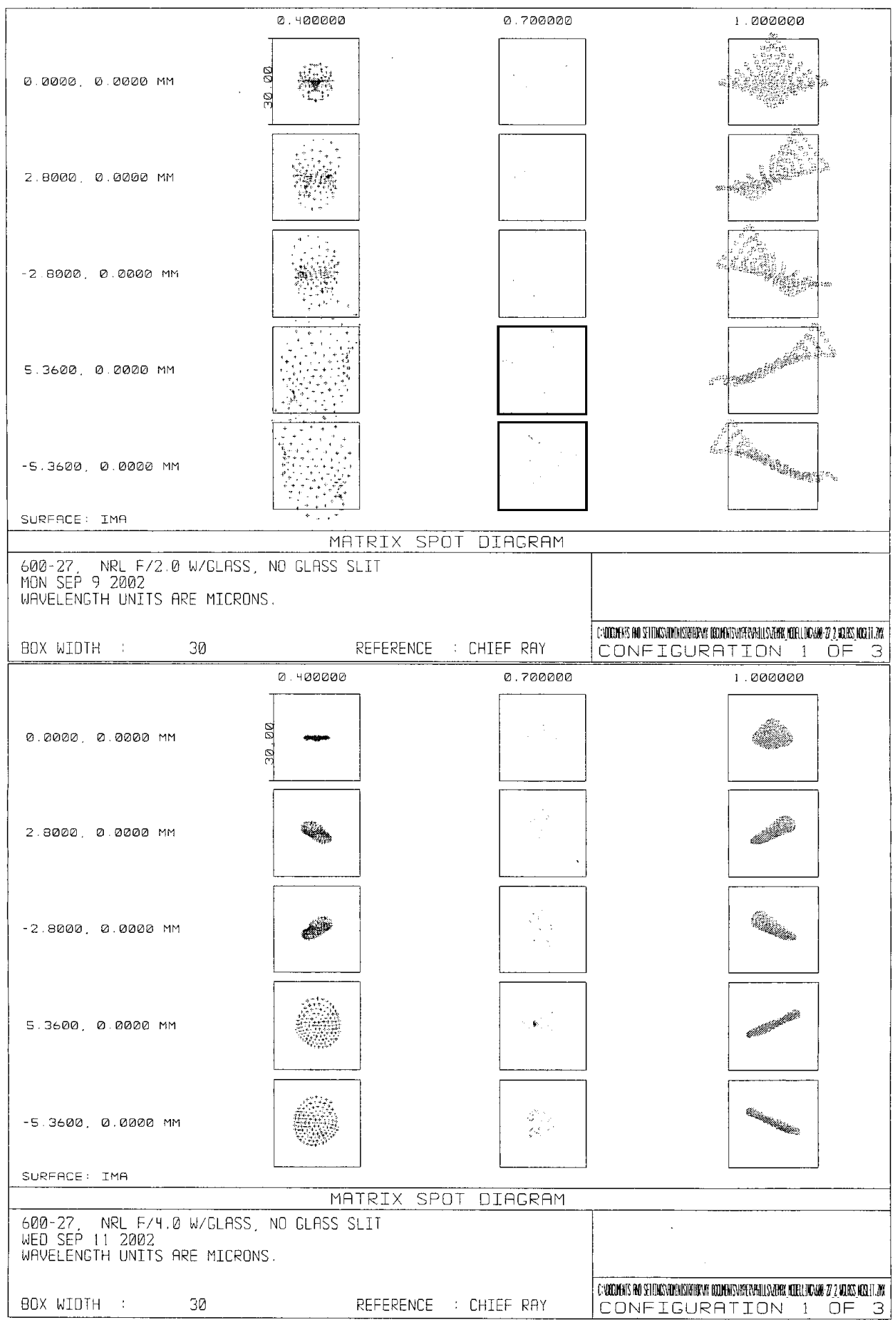

Figure 3. Spot sizes modeled using Zemax ${ }^{\mathrm{TM}}$ for $\mathrm{F} / 2.0$ (top) and F/4.0 (bottom) for the field angles and wavelengths shown on table 1. Modeling is the same as table 1 . Note that the width of each box is 30 microns. 
Figure 3 illustrates the calculations shown in table 1, again illustrating that at F/2.0 the spot sizes are large compared with the 12 micron pixel size of the array. It is also evident that the shorter wavelengths are more sensitive to the field angle than the longer wavelengths.

\section{Time Tagging}

For proper geolocation, the data frames must be temporally matched to the GPS/INS data to within a few milliseconds, depending on frame rate. Before 2001 the PHLLS data frames were time tagged using the Windows NT4.0 operating system clock. As NT4.0 is not specifically tailored to handle only our hardware tasking, the process of grabbing the system time is sometimes interrupted by other tasks. This lead to a random temporal latency between the time an individual frame was acquired and the time for which it was tagged, sometimes on the order of seconds. This was effectively useless for proper geolocation and it had to be performed using ground control points during post-processing. Starting with the LEO-15 2001 deployment this was fixed by using a "true time card" which uses a GPS system to grab the time. This time is then stored in a registry and can be grabbed with the GPS information whenever the operating system allows and can be placed in the acquired frames. Figure 4 is an illustration of the fidelity of our current geolocation procedure.
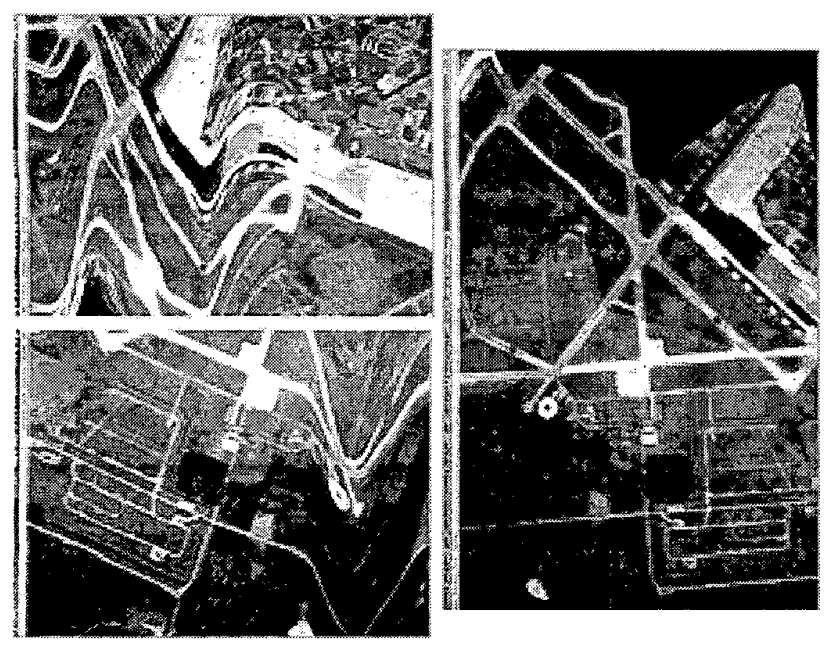

Figure 4. Left- RGB image of raw PHILLS data and rightgeorectified image. The data was collected over MacDill Air Force Base, Tampa FL, in 2002.

\section{Conclusions (deployments)}

The current incarnation of the ocean PHILLS has been deployed in the field in January of 2003. The results of this deployment will be given at a later date, with an emphasis on scattered light, signal to noise ratio, and focusing. Additional laboratory characterization will be performed and reported. It is expected that the effect of scatter at the longer wavelengths should be reduced due to the better masking.

\section{REFERENCES}

[1] J. Bowles, M. Kappus, J. Antoniades, M. Baumback, M Czarnaski, C. O. Davis and J. Grossmann, "Calibration of inexpensive pushbroom imaging spectrometers", Metrologica 35:, 657-661, 1998.

[2] Curtiss O. Davis, Jeffrey Bowles, Robert A. Leathers, Dan Korwan, T. Valerie Downes, William A. Snyder, W. Joe Rhea, Wei Chen, "Ocean PHILLS hyperspectral imager: design, characterization, and calibration", Optics Express 10:4, 210-221, 2002.

Dr. Daniel R. Korwan is a Research Physicist in the Sensor Concepts and Development Section of the Remote Sensing division at the Naval Research Laboratory in Washington, DC. He received his Bachelors of Science in Optics from the University of Rochester in 1984, and his

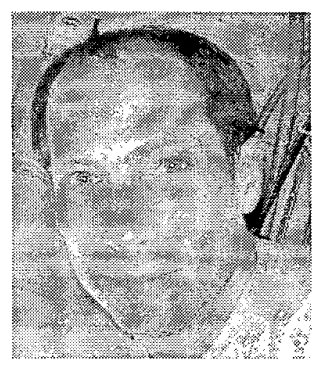

Ph.D. in Physics from Virginia Tech in 1996. Since 1996, he has been an instrument scientist at the Naval Research Laboratory working on space and airborne detectors including Polar Ozone and Aerosol Monitor (POAM II and III), The Navy Earth Map Observer (NEMO), and the ocean PHILLS.

Dr. Jeffrey Bowles received a Ph.D. in physics from the University of California at Irvine in 1993. His primary research area is that of optical remote sensing. His work involves development of hyperspectral, and other, imaging systems deployed from aircraft and methods to analyze the data such as ORASIS.

Dr Curtiss O. Davis is Senior Scientist for Optical Remote Sensing and Head of the Optical Sensing Section in the Remote Sensing Division of the Naval Research Laboratory in Washington, D.C. He received his Bachelors of Science in Zoology from the University of California at Berkeley in 1966 and his Ph.D. in Oceanography

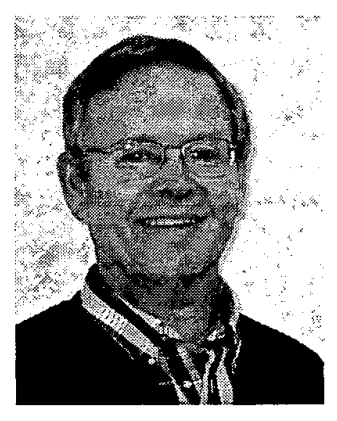


Vol. 4-1861

from the University of Washington in 1973. He was Program Manager for the NASA Ocean Productivity Program, in 1984-86. He was a Research Oceanographer and Group Supervisor, of the Biological and Polar Oceanography at the Jet Propulsion Laboratory from Dec 1986 - Mar 1994. While at JPL he was the Project Scientist, for the High Resolution Imaging Spectrometer (HIRIS), July 1990 - Mar 1994. In 1994 he moved to NRL to develop a program in hyperspectral remote sensing of the coastal ocean. He is a member of the NASA SeaWiFS Science Team and has participated in numerous calibration and validation experiments. He is the Project Scientist for the Navy's Hyperspectral Remote Sensing Technology (HRST) Program, a focused effort to develop the use of hyperspectral imaging for the characterization of the coastal ocean and to fly the Coastal Ocean Imaging Spectrometer (COIS) in space. 